International Journal on Cybernetics \& Informatics (IJCI) Vol. 4, No. 1, February 2015

\title{
Some Results on Fuzzy Soft Multi Sets
}

\author{
Anjan Mukherjee ${ }^{1}$ and Ajoy Kanti Das ${ }^{2}$ \\ ${ }^{1}$ Department of Mathematics, Tripura University, Agartala-799022,Tripura, INDIA. \\ ${ }^{2}$ Department of Mathematics, ICV-College, Belonia -799155, Tripura, INDIA.
}

\begin{abstract}
In this paper we define some new operations in fuzzy soft multi set theory and show that the De Morgan's type of results hold in fuzzy soft multi set theory with respect to these newly defined operations in our way. Also some new results along with illustrating examples have been put forward in our work.
\end{abstract}

\section{KEYWORDS}

Fuzzy set, soft set, soft multi set, fuzzy soft multi set.

\section{INTRODUCTION}

Theory of fuzzy sets, soft sets and soft multi sets are powerful mathematical tools for modeling various types of vagueness and uncertainty. In 1999, Molodtsov [9] initiated soft set theory as a completely generic mathematical tool for modelling uncertainty and vague concepts. Later on Maji et al. [8] presented some new definitions on soft sets and discussed in details the application of soft set in decision making problem. Based on the analysis of several operations on soft sets introduced in [9], Ali et al. [2] presented some new algebraic operations for soft sets and proved that certain De Morgan's law holds in soft set theory with respect to these new definitions. Combining soft sets [9] with fuzzy sets [13], Maji et al. [7] defined fuzzy soft sets, which are rich potential for solving decision making problems. Alkhazaleh and others $[1,3,5,6,12]$ as a generalization of Molodtsov's soft set, presented the definition of a soft multi set and its basic operations such as complement, union, and intersection etc. In 2012, Alkhazaleh and Salleh [4] introduced the concept of fuzzy soft multi set theory and studied the application of these sets and recently, Mukherjee and Das $[10,11]$ constructed the fundamental theory on soft multi topological spaces and fuzzy soft multi topological spaces.

In this paper we define some new notions in fuzzy soft multi set theory and show that the De Morgan's type of results holds in fuzzy soft multi set theory for the newly defined operations in our way. Also some new results along with illustrating examples have been put forward in our work.

\section{PRELIMINARY NOTES}

In this section, we recall some basic notions in soft set theory, and fuzzy soft multi set theory. Molodstov defined soft set in the following way. Let $\mathrm{U}$ be an initial universe and $\mathrm{E}$ be a set of parameters. Let $P(U)$ denotes the power set of $\mathrm{U}$ and $\mathrm{A} \subseteq \mathrm{E}$.

\section{Definition 2.1[9]}

A pair $(F, A)$ is called a soft set over $\mathrm{U}$, where $F$ is a mapping given by $F: A \rightarrow P(U)$. In other words, soft set over $\mathrm{U}$ is a parameterized family of subsets of the universe $\mathrm{U}$. 


\section{Definition 2.2 [7]}

Let $\mathrm{U}$ be an initial universe and $\mathrm{E}$ be a set of parameters. Let $\mathrm{F}(\mathrm{U})$ be the set of all fuzzy subsets of $\mathrm{U}$ and $A \subseteq E$. Then the pair $(\mathrm{F}, \mathrm{A})$ is called a fuzzy soft set over $\mathrm{U}$, where $\mathrm{F}$ is a mapping given by $\mathrm{F}: \mathrm{A} \rightarrow \mathrm{F}(\mathrm{U})$.

\section{Definition 2.3[4]}

Let $\left\{U_{i}: i \in I\right\}$ be a collection of universes such that $\bigcap_{i \in I} U_{i}=\phi$ and let $\left\{E_{U_{i}}: i \in I\right\}$ be a collection of sets of parameters. Let $U=\prod_{i \in I} F S\left(U_{i}\right)$ where $F S\left(U_{i}\right)$ denotes the set of all fuzzy subsets of $U_{i}, E=\prod_{i \in I} E_{U_{i}}$ and $A \subseteq E$. A pair (F, A) is called a fuzzy soft multi set over $\mathrm{U}$, where $F: A \rightarrow U$ is a mapping given by $\forall e \in A$,

$$
F(e)=\left(\left\{\frac{u}{\mu_{F(e)}(u)}: u \in U_{i}\right\}: i \in I\right)
$$

\section{Definition 2.4[12]}

The complement of a fuzzy soft multi set $(F, A)$ over $\mathrm{U}$ is denoted by $(F, A)^{c}$ and is defined by $(F, A)^{c}=\left(F^{c}, A\right)$, where $\forall e \in A$

$$
F^{c}(e)=\left(\left\{\frac{u}{1-\mu_{F(e)}(u)}: u \in U_{i}\right\}: i \in I\right) .
$$

\section{A STUDY ON SOME OPERATIONS IN FUZZY SOFT MULTI SETS}

\section{Definition 3.1}

A fuzzy soft multi set (F, A) over $U$ is called fuzzy soft multi subset of a fuzzy soft multi set (G, B) if

(a) $A \subseteq B$ and

(b) $\forall e \in A, F(e) \subseteq G(e) \Leftrightarrow \mu_{F(e)}(u) \leq \mu_{G(e)}(u), \forall u \in U_{i}, i \in I$.

This relationship is denoted by $(\mathrm{F}, \mathrm{A}) \tilde{\subseteq}(\mathrm{G}, \mathrm{B})$.

\section{Definition 3.2}

Two fuzzy soft multiset $(F, A)$ and $(G, B)$ over $\mathrm{U}$ is called equal if $(F, A)$ is fuzzy soft multi subset of $(G, B)$ and $(G, B)$ is fuzzy soft multi subset of $(F, A)$.

\section{Definition 3.3}

A fuzzy soft multiset $(F, A)$ over $\mathrm{U}$ is called a null fuzzy soft multiset, denoted by $(F, A)_{\varphi}$, if all the fuzzy soft multiset parts of $(F, A)$ equals $\phi$. 


\section{Definition 3.4}

A fuzzy soft multiset $(\mathrm{F}, \mathrm{A})$ over $\mathrm{U}$ is called an absolute fuzzy soft multiset, denoted by $(F, A)_{U}$, if $\left(e_{U_{i}, j}, F_{e_{U_{i}, j}}\right)=U_{i}, \forall i$.

\section{Definition 3.5}

Union of two fuzzy soft multisets $(F, A)$ and $(G, B)$ over $U$ is a fuzzy soft multiset $(H, D)$ where $D=A \cup B$ and $\forall e \in D$,

$$
H(e)= \begin{cases}F(e), & \text { if } e \in A-B \\ G(e), & \text { if } e \in B-A \\ \cup(F(e), G(e)), & \text { if } e \in A \cap B\end{cases}
$$

Where $U(F(e), G(e))=s\left(F_{e_{U_{i}, j}}, G_{e_{U_{i}, j}}\right) \forall i \in\{1,2,3, \ldots, \mathrm{n}\}$ and $j \in\{1,2,3, . ., \mathrm{n}\}$ with $\mathrm{s}$ as an snorm and is written as $(F, A) \tilde{\cup}(G, B)=(H, D)$

\section{Proposition 3.6}

If $(\mathrm{F}, \mathrm{A}),(\mathrm{G}, \mathrm{B})$ and $(\mathrm{H}, \mathrm{D})$ are three fuzzy soft multisets over $\mathrm{U}$, then

(a) $(F, A) \tilde{\cup}((G, B) \tilde{\cup}(H, D))=((F, A) \tilde{\cup}(G, B)) \tilde{\cup}(H, D)$,

(b) $(F, A) \tilde{\cup}(F, A)=(F, A)$,

(c) $(F, A) \tilde{\cup}(G, A)_{\varphi}=(F, A)$,

(d) $(F, A) \tilde{\cup}(G, A)_{U}=(G, A)_{U}$,

\section{Definition 3.7}

Intersection of two fuzzy soft multisets $(\mathrm{F}, A)$ and $(\mathrm{G}, B)$ over $U$ is a fuzzy soft multiset $(\mathrm{H}, \mathrm{D})$ where $D=A \cap B$ and $\forall e \in D$,

$$
H(e)= \begin{cases}F(e), & \text { if } e \in A-B \\ G(e), & \text { if } e \in B-A \\ \cap(F(e), G(e)), & \text { if } e \in A \cap B\end{cases}
$$

where $\bigcap(F(e), G(e))=t\left(F_{e_{U_{i}, j}}, G_{e_{U_{i}, j}}\right) \forall i \in\{1,2,3, \ldots, \mathrm{n}\}$ and $j \in\{1,2,3, \ldots, \mathrm{n}\}$ with $\mathrm{t}$ as an $\mathrm{t}$ norm and is written as $(F, A) \tilde{\cup}(G, B)=(H, C)$

\section{Proposition 3.8}

If $(F, A),(G, B)$ and $(H, D)$ are three fuzzy soft multisets over $\mathrm{U}$, then

(a) $(F, A) \tilde{\cap}((G, B) \tilde{\cap}(H, D))=((F, A) \tilde{\cap}(G, B)) \tilde{\cap}(H, D)$,

(b) $(F, A) \tilde{\cap}(F, A)=(F, A)$,

(c) $(F, A) \tilde{\cap}(G, A)_{\varphi}=(G, A)_{\varphi}$,

(d) $(F, A) \tilde{\cap}(G, A)_{U}=(F, A)$, 
(e) $(F, A) \tilde{\cap}(G, A)_{U}=(F, A)$,

\section{Definition3.9}

The complement of fuzzy soft multiset $(F, A)$ over $\mathrm{U}$ is denoted by $(F, A)^{c}$ and is defined by $(F, A)^{c}=\left(F^{c}, A\right)$, where $F^{c}: A \rightarrow U$ is a mapping given by $F^{c}(\alpha)=c(F(\alpha)), \forall \alpha \in A$, where $\mathrm{c}$ is fuzzy complement.

\section{Proposition 3.10}

For a fuzzy soft multiset $(F, A)$ over $\mathrm{U}$,

(a) $\left((F, A)^{c}\right)^{c}=(F, A)$,

(b) $(F, A)_{\approx \varphi_{i}}^{c}=(F, A)_{\approx U_{i}}$,

(c) $(F, A)_{\varphi}^{c}=(F, A)_{U}$,

(d) $(F, A)_{\approx U_{i}}^{c}=(F, A)_{\approx \varphi_{i}}$,

(e) $(F, A)_{U}^{c}=(F, A)_{\varphi}$,

Now we introduce some new types of operations

\section{Definition 3.11}

The restricted union of two fuzzy soft multi sets $(\mathrm{F}, \mathrm{A})$ and $(\mathrm{G}, \mathrm{B})$ over $\mathrm{U}$ is a fuzzy soft multi set $(\mathrm{H}, \mathrm{C}) \quad$ where $C=A \cap B \quad$ and $\quad \forall e \in C, \quad H(e)=\bigcup(F(e), G(e))$, $=\left(\left\{\frac{u}{\max \left\{\mu_{F(e)}(u), \mu_{G(e)}(u)\right\}}: u \in U_{i}\right\}: i \in I\right)$ and is written as $(F, A) \tilde{\cup}_{R}(G, B)=(H, C)$.

\section{Definition 3.12}

The extended union of two fuzzy soft multi sets $(F, A)$ and $(G, B)$ over $\mathrm{U}$ is a fuzzy soft multi set $(H, D)$, where $D=A \cup B$ and $\forall e \in D$,

$$
H(e)= \begin{cases}F(e), & \text { if } e \in A-B \\ G(e), & \text { if } e \in B-A \\ \cup(F(e), G(e)), & \text { if } e \in A \cap B\end{cases}
$$

where $\quad U(F(e), G(e))=\left(\left\{\frac{u}{\max \left\{\mu_{F(e)}(u), \mu_{G(e)}(u)\right\}}: u \in U_{i}\right\}: i \in I\right) \quad$ and $\quad$ is written as $(F, A) \tilde{\cup}_{E}(G, B)=(H, D)$.

\section{Definition 3.13}


International Journal on Cybernetics \& Informatics (IJCI) Vol. 4, No. 1, February 2015

The restricted intersection of two intuitionistic fuzzy soft multi sets $(F, A)$ and $(G, B)$ over $\mathrm{U}$ is a fuzzy soft multi set $(H, D)$ where $D=A \cap B$ and $\forall e \in D, \quad H(e)=\bigcap(F(e), G(e))$ $=\left(\left\{\frac{u}{\min \left\{\mu_{F(e)}(u), \mu_{G(e)}(u)\right\}}: u \in U_{i}\right\}: i \in I\right)$ and is written as $(F, A) \tilde{\cap}_{R}(G, B)=(H, D)$.

\section{Definition 3.14}

The extended intersection of two fuzzy soft multi sets $(F, A)$ and $(G, B)$ over $\mathrm{U}$ is a fuzzy soft multi set $(H, D)$, where $D=A \cup B$ and $\forall e \in D$,

$$
H(e)= \begin{cases}F(e), & \text { if } e \in A-B \\ G(e), & \text { if } e \in B-A \\ \cap(F(e), G(e)), & \text { if } e \in A \cap B\end{cases}
$$

where $\cap(F(e), G(e))=\left(\left\{\frac{u}{\min \left\{\mu_{F(e)}(u), \mu_{G(e)}(u)\right\}}: u \in U_{i}\right\}: i \in I\right)$ and is written as $(F, A) \tilde{\cap}_{E}(G, B)=(H, D)$.

\section{Example 3.15}

Let us consider there are two universes $U_{1}=\left\{h_{1}, h_{2}, h_{3}\right\}, U_{2}=\left\{c_{1}, c_{2}\right\}$ and let $\left\{E_{U_{1}}, E_{U_{2}}\right\}$ be a collection of sets of decision parameters related to the above universes, where

$E_{U_{1}}=\left\{e_{U_{1}, 1}=\exp\right.$ ensive, $e_{U_{1}, 2}=$ cheap, $e_{U_{1}, 3}=$ wooden $\}$

$E_{U_{1}}=\left\{e_{U_{2}, 1}=\exp\right.$ ensive, $e_{U_{2}, 2}=$ cheap, $e_{U_{2}, 3}=$ sporty $\}$,

Let $U=\prod_{i=1}^{2} F S\left(U_{i}\right), E=\prod_{i=1}^{2} E_{U_{i}}$ and

$$
\begin{gathered}
A=\left\{e_{1}=\left(e_{U_{1}, 1}, e_{U_{2}, 1}\right), e_{2}=\left(e_{U_{1}, 1}, e_{U_{2}, 2}\right), e_{3}=\left(e_{U_{1}, 2}, e_{U_{2}, 1}\right)\right\}, \\
B=\left\{e_{1}=\left(e_{U_{1}, 1}, e_{U_{2}, 1}\right), e_{2}=\left(e_{U_{1}, 1}, e_{U_{2}, 2}\right), e_{4}=\left(e_{U_{1}, 3}, e_{U_{2}, 2}\right)\right\} .
\end{gathered}
$$

Suppose that

$$
\begin{aligned}
& (F, A)=\left\{\left(e_{1},\left(\left\{\frac{h_{1}}{0.2}, \frac{h_{2}}{0.4}, \frac{h_{3}}{0.8}\right\},\left\{\frac{c_{1}}{0.8}, \frac{c_{2}}{0.5}\right\}\right)\right),\left(e_{2},\left(\left\{\frac{h_{1}}{0.7}, \frac{h_{2}}{0.7}, \frac{h_{3}}{1}\right\},\left\{\frac{c_{1}}{0.8}, \frac{c_{2}}{0.6}\right\}\right)\right),\right. \\
& \left.\left(e_{3},\left(\left\{\frac{h_{1}}{0.9}, \frac{h_{2}}{0.7}, \frac{h_{3}}{1}\right\},\left\{\frac{c_{1}}{0.3}, \frac{c_{2}}{0.5}\right\}\right)\right)\right\}, \\
& (G, B)=\left\{\left(e_{1},\left(\left\{\frac{h_{1}}{0.3}, \frac{h_{2}}{0.3}, \frac{h_{3}}{0.7}\right\},\left\{\frac{c_{1}}{0.7}, \frac{c_{2}}{0.6}\right\}\right)\right),\left(e_{2},\left(\left\{\frac{h_{1}}{0.5}, \frac{h_{2}}{0.8}, \frac{h_{3}}{1}\right\},\left\{\frac{c_{1}}{0.4}, \frac{c_{2}}{0.6}\right\}\right)\right),\right. \\
& \left.\left(e_{4},\left(\left\{\frac{h_{1}}{0.8}, \frac{h_{2}}{0.9}, \frac{h_{3}}{1}\right\},\left\{\frac{c_{1}}{0.5}, \frac{c_{2}}{0.2}\right\}\right)\right)\right\},
\end{aligned}
$$

then we have, 
International Journal on Cybernetics \& Informatics (IJCI) Vol. 4, No. 1, February 2015

(i). $(F, A) \tilde{\cup}_{R}(G, B)=\left\{\left(e_{1},\left(\left\{\frac{h_{1}}{0.3}, \frac{h_{2}}{0.4}, \frac{h_{3}}{0.8}\right\},\left\{\frac{c_{1}}{0.8}, \frac{c_{2}}{0.6}\right\}\right)\right),\left(e_{2},\left(\left\{\frac{h_{1}}{0.7}, \frac{h_{2}}{0.8}, \frac{h_{3}}{1}\right\},\left\{\frac{c_{1}}{0.8}, \frac{c_{2}}{0.6}\right\}\right)\right)\right\}$

(ii). $(F, A) \tilde{\cup}_{E}(G, B)=\left\{\left(e_{1},\left(\left\{\frac{h_{1}}{0.3}, \frac{h_{2}}{0.4}, \frac{h_{3}}{0.8}\right\},\left\{\frac{c_{1}}{0.8}, \frac{c_{2}}{0.6}\right\}\right)\right),\left(e_{2},\left(\left\{\frac{h_{1}}{0.7}, \frac{h_{2}}{0.8}, \frac{h_{3}}{1}\right\},\left\{\frac{c_{1}}{0.8}, \frac{c_{2}}{0.6}\right\}\right)\right)\right.$,

$$
\left.\left(e_{3},\left(\left\{\frac{h_{1}}{0.9}, \frac{h_{2}}{0.7}, \frac{h_{3}}{1}\right\},\left\{\frac{c_{1}}{0.3}, \frac{c_{2}}{0.5}\right\}\right)\right),\left(e_{4},\left(\left\{\frac{h_{1}}{0.8}, \frac{h_{2}}{0.9}, \frac{h_{3}}{1}\right\},\left\{\frac{c_{1}}{0.5}, \frac{c_{2}}{0.2}\right\}\right)\right)\right\},
$$

(iii). $(F, A) \tilde{\cap}_{R}(G, B)=\left\{\left(e_{1},\left(\left\{\frac{h_{1}}{0.2}, \frac{h_{2}}{0.3}, \frac{h_{3}}{0.7}\right\},\left\{\frac{c_{1}}{0.7}, \frac{c_{2}}{0.5}\right\}\right)\right),\left(e_{2},\left(\left\{\frac{h_{1}}{0.5}, \frac{h_{2}}{0.7}, \frac{h_{3}}{1}\right\},\left\{\frac{c_{1}}{0.4}, \frac{c_{2}}{0.6}\right\}\right)\right)\right\}$

(iv). $(F, A) \tilde{\cap}_{E}(G, B)=\left\{\left(e_{1},\left(\left\{\frac{h_{1}}{0.2}, \frac{h_{2}}{0.3}, \frac{h_{3}}{0.7}\right\},\left\{\frac{c_{1}}{0.7}, \frac{c_{2}}{0.5}\right\}\right)\right),\left(e_{2},\left(\left\{\frac{h_{1}}{0.5}, \frac{h_{2}}{0.7}, \frac{h_{3}}{1}\right\},\left\{\frac{c_{1}}{0.4}, \frac{c_{2}}{0.6}\right\}\right)\right)\right.$

$$
\left.\left(e_{3},\left(\left\{\frac{h_{1}}{0.9}, \frac{h_{2}}{0.7}, \frac{h_{3}}{1}\right\},\left\{\frac{c_{1}}{0.3}, \frac{c_{2}}{0.5}\right\}\right)\right),\left(e_{4},\left(\left\{\frac{h_{1}}{0.8}, \frac{h_{2}}{0.9}, \frac{h_{3}}{1}\right\},\left\{\frac{c_{1}}{0.5}, \frac{c_{2}}{0.2}\right\}\right)\right)\right\} \text {, }
$$

\section{Proposition 3.16}

For two fuzzy soft multi sets $(F, A)$ and $(G, B)$ over $\mathrm{U}$, then we have

1. $\left((F, A) \tilde{\cup}_{E}(G, B)\right)^{c} \tilde{\subseteq}(F, A)^{c} \tilde{\cup}_{E}(G, B)^{c}$

2. $(F, A)^{c} \tilde{\cap}_{E}(G, B)^{c} \tilde{\subseteq}\left((F, A) \tilde{\cap}_{E}(G, B)\right)^{c}$

\section{Proof.}

1. Let $(F, A) \tilde{\cup}_{E}(G, B)=(H, C)$, where $C=A \cup B$ and $\forall e \in C$,

$H(e)= \begin{cases}F(e), & \text { if } e \in A-B \\ G(e), & \text { if } e \in B-A \\ \cup(F(e), G(e)), & \text { if } e \in A \cap B,\end{cases}$

where $\bigcup(F(e), G(e))=\left(\left\{\frac{u}{\max \left\{\mu_{F(e)}(u), \mu_{G(e)}(u)\right\}}: u \in U_{i}\right\}: i \in I\right)$

Thus $\left((F, A) \tilde{\cup}_{E}(G, B)\right)^{c}=(H, C)^{c}=\left(H^{c}, C\right)$, where $C=A \cup B$ and $\forall e \in C$,

$$
H^{c}(e)= \begin{cases}F^{c}(e), & \text { if } e \in A-B \\ G^{c}(e), & \text { if } e \in B-A \\ (\bigcup(F(e), G(e)))^{c}, & \text { if } e \in A \cap B,\end{cases}
$$

where $(\bigcup(F(e), G(e)))^{c}=\left(\left\{\frac{u}{1-\max \left\{\mu_{F(e)}(u), \mu_{G(e)}(u)\right\}}: u \in U_{i}\right\}: i \in I\right)$

$$
=\left(\left\{\frac{u}{\min \left\{1-\mu_{F(e)}(u), 1-\mu_{G(e)}(u)\right\}}: u \in U_{i}\right\}: i \in I\right)
$$

Again, $(F, A)^{c} \tilde{\cup}_{E}(G, B)^{c}=\left(F^{c}, A\right) \tilde{\cup}_{E}\left(G^{c}, B\right)=(K, D)$. Where $D=A \cup B$ and $\forall e \in D$,

$$
K(e)= \begin{cases}F^{c}(e), & \text { if } e \in A-B \\ G^{c}(e), & \text { if } e \in B-A \\ \cup\left(F^{c}(e), G^{c}(e)\right), & \text { if } e \in A \cap B,\end{cases}
$$


International Journal on Cybernetics \& Informatics (IJCI) Vol. 4, No. 1, February 2015

where $U\left(F^{c}(e), G^{c}(e)\right)=\left(\left\{\frac{u}{\max \left\{1-\mu_{F(e)}(u), 1-\mu_{G(e)}(u)\right\}}: u \in U_{i}\right\}: i \in I\right)$.

We see that $C=D$ and $\forall e \in C, H^{c}(e) \subseteq K(e)$.

Thus $\left((F, A) \tilde{\cup}_{E}(G, B)\right)^{c} \tilde{\subseteq}(F, A)^{c} \tilde{\cup}_{E}(G, B)^{c}$.

2. Let $(F, A) \tilde{\cap}_{E}(G, B)=(H, C)$, where $C=A \cup B$ and $\forall e \in C$,

$H(e)= \begin{cases}F(e), & \text { if } e \in A-B \\ G(e), & \text { if } e \in B-A \\ \cap(F(e), G(e)), & \text { if } e \in A \cap B,\end{cases}$

where $\cap(F(e), G(e))=\left(\left\{\frac{u}{\min \left\{\mu_{F(e)}(u), \mu_{G(e)}(u)\right\}}: u \in U_{i}\right\}: i \in I\right)$

Thus $\left((F, A) \tilde{\cap}_{E}(G, B)\right)^{c}=(H, C)^{c}=\left(H^{c}, C\right)$, where $C=A \cup B$ and $\forall e \in C$,

$H^{c}(e)= \begin{cases}F^{c}(e), & \text { if } e \in A-B \\ G^{c}(e), & \text { if } e \in B-A \\ (\cap(F(e), G(e)))^{c}, & \text { if } e \in A \cap B,\end{cases}$

where $(\cap(F(e), G(e)))^{c}=\left(\left\{\frac{u}{1-\min \left\{\mu_{F(e)}(u), \mu_{G(e)}(u)\right\}}: u \in U_{i}\right\}: i \in I\right)$

$$
=\left(\left\{\frac{u}{\max \left\{1-\mu_{F(e)}(u), 1-\mu_{G(e)}(u)\right\}}: u \in U_{i}\right\}: i \in I\right)
$$

Again, $(F, A)^{c} \tilde{\cap}_{E}(G, B)^{c}=\left(F^{c}, A\right) \tilde{\cap}_{E}\left(G^{c}, B\right)=(K, D)$. Where $D=A \cup B$ and $\forall e \in D$,

$K(e)= \begin{cases}F^{c}(e), & \text { if } e \in A-B \\ G^{c}(e), & \text { if } e \in B-A \\ \cap\left(F^{c}(e), G^{c}(e)\right), & \text { if } e \in A \cap B,\end{cases}$

where $\cap\left(F^{c}(e), G^{c}(e)\right)=\left(\left\{\frac{u}{\min \left\{1-\mu_{F(e)}(u), 1-\mu_{G(e)}(u)\right\}}: u \in U_{i}\right\}: i \in I\right)$.

We see that $C=D$ and $\forall e \in C, K(e) \subseteq H^{c}(e)$.

Thus $(F, A)^{c} \tilde{\cap}_{E}(G, B)^{c} \tilde{\subseteq}\left((F, A) \tilde{\cap}_{E}(G, B)\right)^{c}$.

\section{Proposition 3.17}

For two fuzzy soft multi sets $(F, A)$ and $(G, B)$ over $\mathrm{U}$, then we have

1. $\left((F, A) \tilde{\cup}_{R}(G, B)\right)^{c} \tilde{\subseteq}(F, A)^{c} \tilde{\cup}_{R}(G, B)^{c}$

2. $(F, A)^{c} \tilde{\cap}_{R}(G, B)^{c} \subseteq\left((F, A) \tilde{\cap}_{R}(G, B)\right)^{c}$

\section{Proof.}


1. Let $(F, A) \tilde{\cup}_{R}(G, B)=(H, C)$, where $C=A \cap B$ and $\forall e \in C$,

$H(e)=\bigcup(F(e), G(e))=\left(\left\{\frac{u}{\max \left\{\mu_{F(e)}(u), \mu_{G(e)}(u)\right\}}: u \in U_{i}\right\}: i \in I\right)$.

Thus $\left((F, A) \tilde{\cup}_{R}(G, B)\right)^{c}=(H, C)^{c}=\left(H^{c}, C\right)$, where $C=A \cap B$ and $\forall e \in C$,

$$
\begin{aligned}
H^{c}(e)=(\bigcup(F(e), G(e)))^{c} & =\left\{\left\{\frac{u}{1-\max \left\{\mu_{F(e)}(u), \mu_{G(e)}(u)\right\}}: u \in U_{i}\right\}: i \in I\right) \\
& =\left(\left\{\frac{u}{\min \left\{1-\mu_{F(e)}(u), 1-\mu_{G(e)}(u)\right\}}: u \in U_{i}\right\}: i \in I\right)
\end{aligned}
$$

Again, $(F, A)^{c} \tilde{\cup}_{R}(G, B)^{c}=\left(F^{c}, A\right) \tilde{\cup}_{R}\left(G^{c}, B\right)=(K, D)$. Where $D=A \cap B$ and $\forall e \in D$,

$$
K(e)=\bigcup\left(F^{c}(e), G^{c}(e)\right)=\left(\left\{\frac{u}{\max \left\{1-\mu_{F(e)}(u), 1-\mu_{G(e)}(u)\right\}}: u \in U_{i}\right\}: i \in I\right) \text {. }
$$

We see that $C=D$ and $\forall e \in C, H^{c}(e) \subseteq K(e)$.

$\operatorname{Thus}\left((F, A) \tilde{\cup}_{R}(G, B)\right)^{c} \tilde{\subseteq}(F, A)^{c} \tilde{\cup}_{R}(G, B)^{c}$.

2. Let $(F, A) \tilde{\cap}_{R}(G, B)=(H, C)$, where $C=A \cap B$ and $\forall e \in C$,

$$
H(e)=\bigcap(F(e), G(e))=\left(\left\{\frac{u}{\min \left\{\mu_{F(e)}(u), \mu_{G(e)}(u)\right\}}: u \in U_{i}\right\}: i \in I\right) .
$$

Thus $\left((F, A) \tilde{\cap}_{R}(G, B)\right)^{c}=(H, C)^{c}=\left(H^{c}, C\right)$, where $C=A \cap B$ and $\forall e \in C$,

$$
\begin{aligned}
H^{c}(e)=(\cap(F(e), G(e)))^{c} & =\left\{\left\{\frac{u}{1-\min \left\{\mu_{F(e)}(u), \mu_{G(e)}(u)\right\}}: u \in U_{i}\right\}: i \in I\right) \\
& =\left(\left\{\frac{u}{\max \left\{1-\mu_{F(e)}(u), 1-\mu_{G(e)}(u)\right\}}: u \in U_{i}\right\}: i \in I\right)
\end{aligned}
$$

Again, $(F, A)^{c} \tilde{\cap}_{R}(G, B)^{c}=\left(F^{c}, A\right) \tilde{\cap}_{R}\left(G^{c}, B\right)=(K, D)$. Where $D=A \cap B$ and $\forall e \in D$,

$$
K(e)=\bigcap\left(F^{c}(e), G^{c}(e)\right)=\left(\left\{\frac{u}{\min \left\{1-\mu_{F(e)}(u), 1-\mu_{G(e)}(u)\right\}}: u \in U_{i}\right\}: i \in I\right) .
$$

We see that $C=D$ and $\forall e \in C, K(e) \subseteq H^{c}(e)$.

Thus $(F, A)^{c} \tilde{\cap}_{R}(G, B)^{c} \subseteq\left((F, A) \tilde{\cap}_{R}(G, B)\right)^{c}$.

\section{Proposition 3.18}

For two fuzzy soft multi sets $(F, A)$ and $(G, B)$ over $\mathrm{U}$, then we have

1. $\left((F, A) \tilde{\cap}_{R}(G, B)\right)^{c} \subseteq(F, A)^{c} \tilde{\cup}_{E}(G, B)^{c}$

2. $(F, A)^{c} \tilde{\cap}_{R}(G, B)^{c} \subseteq\left((F, A) \tilde{\cup}_{E}(G, B)\right)^{c}$

\section{Proof.}

1. Let $(F, A) \tilde{\cap}_{R}(G, B)=(H, C)$, where $C=A \cap B$ and $\forall e \in C$, 
International Journal on Cybernetics \& Informatics (IJCI) Vol. 4, No. 1, February 2015

$$
H(e)=\bigcap(F(e), G(e))=\left(\left\{\frac{u}{\min \left\{\mu_{F(e)}(u), \mu_{G(e)}(u)\right\}}: u \in U_{i}\right\}: i \in I\right)
$$

Thus $\left((F, A) \tilde{\cap}_{R}(G, B)\right)^{c}=(H, C)^{c}=\left(H^{c}, C\right)$, where $C=A \cap B$ and $\forall e \in C$,

$$
\begin{aligned}
H^{c}(e) & =\left(\left\{\frac{u}{1-\min \left\{\mu_{F(e)}(u), \mu_{G(e)}(u)\right\}}: u \in U_{i}\right\}: i \in I\right) \\
& =\left(\left\{\frac{u}{\max \left\{1-\mu_{F(e)}(u), 1-\mu_{G(e)}(u)\right\}}: u \in U_{i}\right\}: i \in I\right)
\end{aligned}
$$

Again, $(F, A)^{c} \tilde{\cup}_{E}(G, B)^{c}=\left(F^{c}, A\right) \tilde{\cup}_{E}\left(G^{c}, B\right)=(K, D)$. Where $D=A \cup B$ and $\forall e \in D$,

$K(e)= \begin{cases}F^{c}(e), & \text { if } e \in A-B \\ G^{c}(e), & \text { if } e \in B-A \\ \cup\left(F^{c}(e), G^{c}(e)\right), & \text { if } e \in A \cap B,\end{cases}$

where, $U\left(F^{c}(e), G^{c}(e)\right)=\left(\left\{\frac{u}{\max \left\{1-\mu_{F(e)}(u), 1-\mu_{G(e)}(u)\right\}}: u \in U_{i}\right\}: i \in I\right)$

We see that $C \subseteq D$ and $\forall e \in C, H^{c}(e)=K(e)$.

Thus $\left((F, A) \tilde{\cap}_{R}(G, B)\right)^{c} \subseteq(F, A)^{c} \tilde{\cup}_{E}(G, B)^{c}$.

2. Let $(F, A)^{c} \tilde{\cap}_{R}(G, B)^{c}=\left(F^{c}, A\right) \tilde{\cap}_{R}\left(G^{c}, B\right)=(H, C)$, where $C=A \cap B$ and $\forall e \in C$,

$$
\begin{aligned}
H(e)=\bigcap\left(F^{c}(e), G^{c}(e)\right) & =\left(\left\{\frac{u}{\min \left\{1-\mu_{F(e)}(u), 1-\mu_{G(e)}(u)\right\}}: u \in U_{i}\right\}: i \in I\right) \\
& =\left(\left\{\frac{u}{1-\max \left\{\mu_{F(e)}(u), \mu_{G(e)}(u)\right\}}: u \in U_{i}\right\}: i \in I\right)
\end{aligned}
$$

Again, let $(F, A) \tilde{\cup}_{E}(G, B)=(K, D)$. Where $D=A \cup B$ and $\forall e \in D$,

$K(e)= \begin{cases}F(e), & \text { if } e \in A-B \\ G(e), & \text { if } e \in B-A \\ \cup(F(e), G(e)), & \text { if } e \in A \cap B,\end{cases}$

where, $U(F(e), G(e))=\left(\left\{\frac{u}{\max \left\{\mu_{F(e)}(u), \mu_{G(e)}(u)\right\}}: u \in U_{i}\right\}: i \in I\right)$

Thus $\left((F, A) \tilde{\cup}_{E}(G, B)\right)^{c}=(K, D)^{c}=\left(K^{c}, D\right)$, where $D=A \cup B$ and $\forall e \in D$,

$$
K^{c}(e)= \begin{cases}F^{c}(e), & \text { if } e \in A-B \\ G^{c}(e), & \text { if } e \in B-A \\ (\cup(F(e), G(e)))^{c}, & \text { if } e \in A \cap B,\end{cases}
$$

where, $(\bigcup(F(e), G(e)))^{c}=\left(\left\{\frac{u}{1-\max \left\{\mu_{F(e)}(u), \mu_{G(e)}(u)\right\}}: u \in U_{i}\right\}: i \in I\right)$

We see that $C \subseteq D$ and $\forall e \in C, H(e)=K^{c}(e)$.

Thus $(F, A)^{c} \tilde{\cap}_{R}(G, B)^{c} \subseteq\left((F, A) \tilde{\cup}_{E}(G, B)\right)^{c}$. 


\section{Proposition 3.19(De Morgan Laws)}

For two fuzzy soft multi sets $(F, A)$ and $(G, B)$ over $\mathrm{U}$, then we have

[1]. $\left((F, A) \tilde{\cup}_{R}(G, B)\right)^{c}=(F, A)^{c} \tilde{\cap}_{R}(G, B)^{c}$

[2]. $\left((F, A) \tilde{\cap}_{R}(G, B)\right)^{c}=(F, A)^{c} \tilde{\cup}_{R}(G, B)^{c}$

\section{Proof.}

[1]. Let $(F, A) \tilde{\cup}_{R}(G, B)=(H, C)$, where $C=A \cap B$ and $\forall e \in C$,

$H(e)=\bigcup(F(e), G(e))=\left(\left\{\frac{u}{\max \left\{\mu_{F(e)}(u), \mu_{G(e)}(u)\right\}}: u \in U_{i}\right\}: i \in I\right)$.

Thus $\left((F, A) \tilde{\cup}_{R}(G, B)\right)^{c}=(H, C)^{c}=\left(H^{c}, C\right)$, where $C=A \cap B$ and $\forall e \in C$,

$$
\begin{aligned}
H^{c}(e) & =\left(\left\{\frac{u}{1-\max \left\{\mu_{F(e)}(u), \mu_{G(e)}(u)\right\}}: u \in U_{i}\right\}: i \in I\right) \\
& =\left(\left\{\frac{u}{\min \left\{1-\mu_{F(e)}(u), 1-\mu_{G(e)}(u)\right\}}: u \in U_{i}\right\}: i \in I\right)
\end{aligned}
$$

Again, $(F, A)^{c} \tilde{\cap}_{R}(G, B)^{c}=\left(F^{c}, A\right) \tilde{\cap}_{R}\left(G^{c}, B\right)=(K, D)$, where $D=A \cap B$ and $\forall e \in D$,

$$
K(e)=\bigcap\left(F^{c}(e), G^{c}(e)\right)=\left(\left\{\frac{u}{\min \left\{1-\mu_{F(e)}(u), 1-\mu_{G(e)}(u)\right\}}: u \in U_{i}\right\}: i \in I\right) .
$$

We see that $C=D$ and $\forall e \in C, H^{c}(e)=K(e)$. Hence proved.

[2]. Let $(F, A) \tilde{\cap}_{R}(G, B)=(H, C)$, where $C=A \cap B$ and $\forall e \in C$,

$$
H(e)=\bigcap(F(e), G(e))=\left(\left\{\frac{u}{\min \left\{\mu_{F(e)}(u), \mu_{G(e)}(u)\right\}}: u \in U_{i}\right\}: i \in I\right) .
$$

Thus $\left((F, A) \tilde{\cap}_{R}(G, B)\right)^{c}=(H, C)^{c}=\left(H^{c}, C\right)$, where $C=A \cap B$ and $\forall e \in C$,

$$
\begin{aligned}
H^{c}(e) & =\left(\left\{\frac{u}{1-\min \left\{\mu_{F(e)}(u), \mu_{G(e)}(u)\right\}}: u \in U_{i}\right\}: i \in I\right) \\
& =\left(\left\{\frac{u}{\max \left\{1-\mu_{F(e)}(u), 1-\mu_{G(e)}(u)\right\}}: u \in U_{i}\right\}: i \in I\right)
\end{aligned}
$$

Again, $(F, A)^{c} \tilde{\cup}_{R}(G, B)^{c}=\left(F^{c}, A\right) \tilde{\cup}_{R}\left(G^{c}, B\right)=(K, D)$, where $D=A \cap B$ and $\forall e \in D$,

$$
K(e)=\bigcup\left(F^{c}(e), G^{c}(e)\right)=\left(\left\{\frac{u}{\max \left\{1-\mu_{F(e)}(u), 1-\mu_{G(e)}(u)\right\}}: u \in U_{i}\right\}: i \in I\right) .
$$

We see that $C=D$ and $\forall e \in C, H^{c}(e)=K(e)$. Hence proved.

\section{Proposition 3.20(De Morgan Laws)}

For two fuzzy soft multi sets $(F, A)$ and $(G, B)$ over $\mathrm{U}$, then we have

1. $\left((F, A) \tilde{\cup}_{E}(G, B)\right)^{c}=(F, A)^{c} \tilde{\cap}_{E}(G, B)^{c}$

2. $\left((F, A) \tilde{\cap}_{E}(G, B)\right)^{c}=(F, A)^{c} \tilde{\cup}_{E}(G, B)^{c}$ 


\section{Proof.}

1. Let $(F, A) \tilde{\cup}_{E}(G, B)=(H, C)$, where $C=A \cup B$ and $\forall e \in C$,

$H(e)= \begin{cases}F(e), & \text { if } e \in A-B \\ G(e), & \text { if } e \in B-A \\ \cup(F(e), G(e)), & \text { if } e \in A \cap B,\end{cases}$

where $\bigcup(F(e), G(e))=\left(\left\{\frac{u}{\max \left\{\mu_{F(e)}(u), \mu_{G(e)}(u)\right\}}: u \in U_{i}\right\}: i \in I\right)$

Thus $\left((F, A) \tilde{\cup}_{E}(G, B)\right)^{c}=(H, C)^{c}=\left(H^{c}, C\right)$, where $C=A \cup B$ and $\forall e \in C$,

$$
\begin{aligned}
& H^{c}(e)= \begin{cases}F^{c}(e), & \text { if } e \in A-B \\
G^{c}(e), & \text { if } e \in B-A \\
(\cup(F(e), G(e)))^{c}, & \text { if } e \in A \cap B,\end{cases} \\
& \text { where }(\bigcup(F(e), G(e)))^{c}=\left(\left\{\frac{u}{1-\max \left\{\mu_{F(e)}(u), \mu_{G(e)}(u)\right\}}: u \in U_{i}\right\}: i \in I\right) \\
&=\left(\left\{\frac{u}{\min \left\{1-\mu_{F(e)}(u), 1-\mu_{G(e)}(u)\right\}}: u \in U_{i}\right\}: i \in I\right)
\end{aligned}
$$

Again, $(F, A)^{c} \tilde{\cap}_{E}(G, B)^{c}=\left(F^{c}, A\right) \tilde{\cap}_{E}\left(G^{c}, B\right)=(K, D)$, where $D=A \cup B$ and $\forall e \in D$,

$K(e)= \begin{cases}F^{c}(e), & \text { if } e \in A-B \\ G^{c}(e), & \text { if } e \in B-A \\ \cap\left(F^{c}(e), G^{c}(e)\right), & \text { if } e \in A \cap B\end{cases}$

where $\cap\left(F^{c}(e), G^{c}(e)\right)=\left(\left\{\frac{u}{\min \left\{1-\mu_{F(e)}(u), 1-\mu_{G(e)}(u)\right\}}: u \in U_{i}\right\}: i \in I\right)$

We see that $C=D$ and $\forall e \in C, H^{c}(e)=K(e)$. Thus the result.

2. Let $(F, A) \tilde{\cap}_{E}(G, B)=(H, C)$, where $C=A \cup B$ and $\forall e \in C$,

$$
H(e)= \begin{cases}F(e), & \text { if } e \in A-B \\ G(e), & \text { if } e \in B-A \\ \cap(F(e), G(e)), & \text { if } e \in A \cap B,\end{cases}
$$

where $\cap(F(e), G(e))=\left(\left\{\frac{u}{\min \left\{\mu_{F(e)}(u), \mu_{G(e)}(u)\right\}}: u \in U_{i}\right\}: i \in I\right)$

Thus $\left((F, A) \tilde{\cap}_{E}(G, B)\right)^{c}=(H, C)^{c}=\left(H^{c}, C\right)$, where $C=A \cup B$ and $\forall e \in C$,

$$
H^{c}(e)= \begin{cases}F^{c}(e), & \text { if } e \in A-B \\ G^{c}(e), & \text { if } e \in B-A \\ (\cap(F(e), G(e)))^{c}, & \text { if } e \in A \cap B,\end{cases}
$$




$$
\text { where } \begin{aligned}
(\cap(F(e), G(e)))^{c} & =\left(\left\{\frac{u}{1-\min \left\{\mu_{F(e)}(u), \mu_{G(e)}(u)\right\}}: u \in U_{i}\right\}: i \in I\right) \\
& =\left(\left\{\frac{u}{\max \left\{1-\mu_{F(e)}(u), 1-\mu_{G(e)}(u)\right\}}: u \in U_{i}\right\}: i \in I\right)
\end{aligned}
$$

Again, $(F, A)^{c} \tilde{\cup}_{E}(G, B)^{c}=\left(F^{c}, A\right) \tilde{\cup}_{E}\left(G^{c}, B\right)=(K, D)$, where $D=A \cup B$ and $\forall e \in D$,

$$
K(e)= \begin{cases}F^{c}(e), & \text { if } e \in A-B \\ G^{c}(e), & \text { if } e \in B-A \\ \cup\left(F^{c}(e), G^{c}(e)\right), & \text { if } e \in A \cap B,\end{cases}
$$

where $U\left(F^{c}(e), G^{c}(e)\right)=\left(\left\{\frac{u}{\max \left\{1-\mu_{F(e)}(u), 1-\mu_{G(e)}(u)\right\}}: u \in U_{i}\right\}: i \in I\right)$

We see that $C=D$ and $\forall e \in C, H^{c}(e)=K(e)$. Hence proved.

\section{Definition 3.21}

If (F, A) and (G, B) be two fuzzy soft multi sets over $\mathrm{U}$, then " $(F, A)$ AND $(G, B)$ " is a fuzzy soft multi set denoted by $(F, A) \wedge(G, B)$ and is defined by $(F, A) \wedge(G, B)=(H, A \times B)$, where $\mathrm{H}$ is mapping given by $\mathrm{H}: \mathrm{A} \times \mathrm{B} \rightarrow \mathrm{U}$ and

$$
\forall(a, b) \in A \times B, H(a, b)=\left(\left\{\frac{u}{\min \left\{\mu_{F(a)}(u), \mu_{G(b)}(u)\right\}}: u \in U_{i}\right\}: i \in I\right) .
$$

\section{Example 3.22}

If we consider two fuzzy soft multi sets $(\mathrm{F}, \mathrm{A})$ and $(\mathrm{G}, \mathrm{B})$ as in example 3.15 , then we have

$$
\begin{aligned}
(F, A) \wedge(G, B)= & \left\{\left(\left(e_{1}, e_{1}\right),\left(\left\{\frac{h_{1}}{0.2}, \frac{h_{2}}{0.3}, \frac{h_{3}}{0.7}\right\},\left\{\frac{c_{1}}{0.7}, \frac{c_{2}}{0.5}\right\}\right)\right),\left(\left(e_{1}, e_{2}\right),\left(\left\{\frac{h_{1}}{0.2}, \frac{h_{2}}{0.4}, \frac{h_{3}}{0.8}\right\},\left\{\frac{c_{1}}{0.4}, \frac{c_{2}}{0.5}\right\}\right)\right),\right. \\
& \left(\left(e_{1}, e_{4}\right),\left(\left\{\frac{h_{1}}{0.2}, \frac{h_{2}}{0.4}, \frac{h_{3}}{0.8}\right\},\left\{\frac{c_{1}}{0.5}, \frac{c_{2}}{0.2}\right\}\right)\right),\left(\left(e_{2}, e_{1}\right),\left(\left\{\frac{h_{1}}{0.3}, \frac{h_{2}}{0.3}, \frac{h_{3}}{0.7}\right\},\left\{\frac{c_{1}}{0.7}, \frac{c_{2}}{0.6}\right\}\right)\right), \\
& \left(\left(e_{2}, e_{2}\right),\left(\left\{\frac{h_{1}}{0.5}, \frac{h_{2}}{0.7}, \frac{h_{3}}{1}\right\},\left\{\frac{c_{1}}{0.4}, \frac{c_{2}}{0.6}\right\}\right)\right),\left(\left(e_{2}, e_{4}\right),\left(\left\{\frac{h_{1}}{0.7}, \frac{h_{2}}{0.7}, \frac{h_{3}}{1}\right\},\left\{\frac{c_{1}}{0.5}, \frac{c_{2}}{0.2}\right\}\right)\right), \\
& \left(\left(e_{3}, e_{1}\right),\left(\left\{\frac{h_{1}}{0.3}, \frac{h_{2}}{0.3}, \frac{h_{3}}{0.7}\right\},\left\{\frac{c_{1}}{0.3}, \frac{c_{2}}{0.5}\right\}\right)\right),\left(\left(e_{3}, e_{2}\right),\left(\left\{\frac{h_{1}}{0.5}, \frac{h_{2}}{0.7}, \frac{h_{3}}{1}\right\},\left\{\frac{c_{1}}{0.3}, \frac{c_{2}}{0.5}\right\}\right)\right), \\
& \left.\left(\left(e_{3}, e_{4}\right),\left(\left\{\frac{h_{1}}{0.8}, \frac{h_{2}}{0.7}, \frac{h_{3}}{1}\right\},\left\{\frac{c_{1}}{0.3}, \frac{c_{2}}{0.2}\right\}\right)\right)\right\}
\end{aligned}
$$

\section{Definition 3.23}

If $(F, A)$ and $(G, B)$ be two fuzzy soft multi sets over $\mathrm{U}$, then " $(F, A)$ OR $(G, B)$ " is a fuzzy soft multi set denoted by $(F, A) \vee(G, B)$ and is defined by $(F, A) \vee(G, B)=(K, A \times B)$, where $\mathrm{K}$ is mapping given by $\mathrm{K}: \mathrm{A} \times \mathrm{B} \rightarrow \mathrm{U}$ and 
International Journal on Cybernetics \& Informatics (IJCI) Vol. 4, No. 1, February 2015

$$
\forall(a, b) \in A \times B, K(a, b)=\left(\left\{\frac{u}{\max \left\{\mu_{F(a)}(u), \mu_{G(b)}(u)\right\}}: u \in U_{i}\right\}: i \in I\right)
$$

\section{Example 3.24}

If we consider two fuzzy soft multi sets $(F, A)$ and $(G, B)$ as in example 3.15 , then we have

$$
\begin{aligned}
(F, A) \vee(G, B)=\{ & \left(\left(e_{1}, e_{1}\right),\left(\left\{\frac{h_{1}}{0.3}, \frac{h_{2}}{0.4}, \frac{h_{3}}{0.8}\right\},\left\{\frac{c_{1}}{0.8}, \frac{c_{2}}{0.6}\right\}\right)\right),\left(\left(e_{1}, e_{2}\right),\left(\left\{\frac{h_{1}}{0.5}, \frac{h_{2}}{0.8}, \frac{h_{3}}{1}\right\},\left\{\frac{c_{1}}{0.8}, \frac{c_{2}}{0.6}\right\}\right)\right), \\
& \left(\left(e_{1}, e_{4}\right),\left(\left\{\frac{h_{1}}{0.8}, \frac{h_{2}}{0.9}, \frac{h_{3}}{1}\right\},\left\{\frac{c_{1}}{0.8}, \frac{c_{2}}{0.5}\right\}\right)\right),\left(\left(e_{2}, e_{1}\right),\left(\left\{\frac{h_{1}}{0.7}, \frac{h_{2}}{0.7}, \frac{h_{3}}{1}\right\},\left\{\frac{c_{1}}{0.8}, \frac{c_{2}}{0.6}\right\}\right)\right), \\
& \left(\left(e_{2}, e_{2}\right),\left(\left\{\frac{h_{1}}{0.7}, \frac{h_{2}}{0.8}, \frac{h_{3}}{1}\right\},\left\{\frac{c_{1}}{0.8}, \frac{c_{2}}{0.6}\right\}\right)\right),\left(\left(e_{2}, e_{4}\right),\left(\left\{\frac{h_{1}}{0.8}, \frac{h_{2}}{0.9}, \frac{h_{3}}{1}\right\},\left\{\frac{c_{1}}{0.8}, \frac{c_{2}}{0.6}\right\}\right)\right), \\
& \left(\left(e_{3}, e_{1}\right),\left(\left\{\frac{h_{1}}{0.9}, \frac{h_{2}}{0.7}, \frac{h_{3}}{1}\right\},\left\{\frac{c_{1}}{0.7}, \frac{c_{2}}{0.6}\right\}\right)\right),\left(\left(e_{3}, e_{2}\right),\left(\left\{\frac{h_{1}}{0.9}, \frac{h_{2}}{0.8}, \frac{h_{3}}{1}\right\},\left\{\frac{c_{1}}{0.4}, \frac{c_{2}}{0.6}\right\}\right)\right), \\
& \left.\left(\left(e_{3}, e_{4}\right),\left(\left\{\frac{h_{1}}{0.9}, \frac{h_{2}}{0.9}, \frac{h_{3}}{1}\right\},\left\{\frac{c_{1}}{0.5}, \frac{c_{2}}{0.5}\right\}\right)\right)\right\}
\end{aligned}
$$

\section{Proposition 3.25}

For two fuzzy soft multi sets $(F, A)$ and $(G, B)$ over $\mathrm{U}$, we have the following

1. $((F, A) \wedge(G, B))^{c}=(F, A)^{c} \vee(G, B)^{c}$

2. $((F, A) \vee(G, B))^{c}=(F, A)^{c} \wedge(G, B)^{c}$

\section{Proof.}

1. Let $(F, A) \wedge(G, B)=(H, A \times B)$, where $\forall a \in A$ and $\forall b \in B$,

$H(a, b)=\bigcap(F(a), G(b))=\left(\left\{\frac{u}{\min \left\{\mu_{F(a)}(u), \mu_{G(b)}(u)\right\}}: u \in U_{i}\right\}: i \in I\right)$

Thus $((F, A) \wedge(G, B))^{c}=(H, A \times B)^{c}=\left(H^{c}, A \times B\right)$, where $\forall(a, b) \in A \times B$,

$$
\begin{aligned}
H^{c}(a, b) & =\left(\left\{\frac{u}{1-\min \left\{\mu_{F(a)}(u), \mu_{G(b)}(u)\right\}}: u \in U_{i}\right\}: i \in I\right) \\
& =\left(\left\{\frac{u}{\max \left\{1-\mu_{F(a)}(u), 1-\mu_{G(b)}(u)\right\}}: u \in U_{i}\right\}: i \in I\right)
\end{aligned}
$$

Again, let $(F, A)^{c} \vee(G, B)^{c}=\left(F^{c}, A\right) \vee\left(G^{c}, B\right)=(K, A \times B)$, where $\forall(a, b) \in A \times B$,

$$
K(a, b)=\bigcup\left(F^{c}(a), G^{c}(b)\right)=\left(\left\{\frac{u}{\max \left\{1-\mu_{F(a)}(u), 1-\mu_{G(b)}(u)\right\}}: u \in U_{i}\right\}: i \in I\right) .
$$

Thus it follows that $((F, A) \wedge(G, B))^{c}=(F, A)^{c} \vee(G, B)^{c}$.

2. Let $(F, A) \vee(G, B)=(H, A \times B)$, where $\forall a \in A$ and $\forall b \in B$,

$$
H(a, b)=\bigcup(F(a), G(b))=\left(\left\{\frac{u}{\max \left\{\mu_{F(a)}(u), \mu_{G(b)}(u)\right\}}: u \in U_{i}\right\}: i \in I\right)
$$


Thus $((F, A) \vee(G, B))^{c}=(H, A \times B)^{c}=\left(H^{c}, A \times B\right)$, where $\forall(a, b) \in A \times B$,

$$
\begin{aligned}
H^{c}(a, b) & =\left(\left\{\frac{u}{1-\max \left\{\mu_{F(a)}(u), \mu_{G(b)}(u)\right\}}: u \in U_{i}\right\}: i \in I\right) \\
& =\left(\left\{\frac{u}{\min \left\{1-\mu_{F(a)}(u), 1-\mu_{G(b)}(u)\right\}}: u \in U_{i}\right\}: i \in I\right)
\end{aligned}
$$

Again, let $(F, A)^{c} \wedge(G, B)^{c}=\left(F^{c}, A\right) \wedge\left(G^{c}, B\right)=(K, A \times B)$, where $\forall(a, b) \in A \times B$,

$$
K(a, b)=\bigcap\left(F^{c}(a), G^{c}(b)\right)=\left(\left\{\frac{u}{\min \left\{1-\mu_{F(a)}(u), 1-\mu_{G(b)}(u)\right\}}: u \in U_{i}\right\}: i \in I\right) .
$$

Thus it follows that $((F, A) \vee(G, B))^{c}=(F, A)^{c} \wedge(G, B)^{c}$.

\section{CONCLUSION}

In this paper, we have made an investigation on existing basic notions and results on fuzzy soft multi sets. Some new results have been stated in our work. Here we shall define some new operations in fuzzy soft multi set theory and show that the De Morgan's type of results holds in fuzzy soft multi set theory with respect to these newly defined operations in our way. These properties may be used in real life problems, like decision making problem, inventory control problem, etc.

\section{REFERENCES}

[1] K. Alhazaymeh \& N. Hassan, (2014) “Vague Soft Multiset Theory”, Int. J. Pure and APPlied MATH., VOL. 93, PP511-523.

[2] M.I. Ali, F. Feng, X. LiU, W.K. Minc \& M. ShabiR, (2009) "ON SOME NEW OPERATiOnS IN SOFT SET THEORY”, COMP. MATH. APPL., VOL. 57, PP1547-1553.

[3] S. Alkhazaleh, A.R. Salleh \& N. Hassan, (2011) Soft Multi Sets Theory, Appl. Math. SCI., VOL. 5, PP3561-3573.

[4] S. Alkhazaleh \& A.R. Salleh, (2012) "FuzZy Soft Multi Sets TheORY", Hindawi Publishing CORPORATION, ABSTRACT AND APPliED ANALYSis, ARTIClE ID 350603, 20 PAGES, DOI: $10.1155 / 2012 / 350603$.

[5] K.V BABITHA \& S.J. John, (2013) “On SOFt Multi SETS”, ANN. FuZZY MATH. INFORM., Vol. 5 PP3544.

[6] H.M. BALAmi \& A. M. Ibrahim, (2013) "SOFT Multiset AND ITS APPLiCATiON IN INFORMATION SYSTEM”, INTERNATIONAL JOURNAL OF SCIENTIFIC RESEARCH AND MANAGEMENT, VOL. 1, PP471-482.

[7] P.K. MAJI, A.R. ROY \& R. BISWAS, (2001) "FuZZY SOFT SETS", J. FUZZY MATH., VOL. 9, PP589-602.

[8] P.K. MAJI, R. BiSWAS \& A.R. ROY, (2003) "SOFT SET THEORY”, COMP. MATH. APPL., Vol. 45 PP555562.

[9] D. Molodtsov, (1999) “Soft SET TheORY-FirSt ReSults”, COMP. MATH. APPL., Vol. 37, PP19-31.

[10] A. MukHERJEe \& A.K. DAS, (2014) "PARAMETERIZED TOPOlOGICAL SPACE INDUCED BY AN Intuitionistic Fuzzy Soft Multi Topological Space”, AnN. Pure AND Applied Math., Vol. 7, PP7-12.

[11] A. MuKHERJEE \& A.K. DAS, (2013) “TOPOlOGICAL STRUCTURE FORMED BY FUZZY SOFT MULTISETS", ReV. Bull. CAL.MATH.SoC., Vol. 21, No.2, PP193-212.

[12] D. Tokat \& I. Osmanoglu, (2011) "Soft MUlti SET AND SOFt MUlti topology", NeVSEHIR UNIVERSITESI FEN BILIMLERI ENSTITUSU DERGISI CILT., VOL. 2, PP109-118. 
[13] L.A. ZADEH, (1965) “FUZZY SETS”, INFORM. CONTROL., VOL. 8, PP338-353.

\begin{abstract}
Authors
Prof. Anjan Mukherjee, Pro Vice Chancellor of Tripura University was born on Jan. 01, 1955 in Kolkata, INDIA. He completed his B.Sc. and M.Sc. in Mathematics from University of Calcutta and obtained his PhD from Tripura University. Prof. Mukherjee has 26 years of research and teaching experience. He published more than 150 research papers in different National and International journals and conference proceedings and has delivered several invited talks. He is also associated with Fuzzy and Rough Sets Association. He had visited University of Texas (U.S.A.), City College of New York (U.S.A.), Malayasia (AMC 5th Asian Mathematical Conference) and Bangladesh.
\end{abstract}

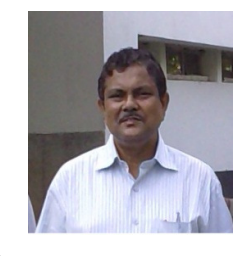

Mr. Ajoy Kanti Das was born on Nov.06, 1987 in Tripura, INDIA. He received the B.Sc (Hons) degree and M.Sc degree in Pure Mathematics from Tripura University, Tripura, INDIA in 2008 and 2010 respectively. He works as a faculty member in the department of Mathematics, ICV-College, Tripura, INDI. His current interest is Topology, Fuzzy Set Theory and Fuzzy Topology, Rough Sets, Soft Set, applications in Fuzzy soft computing, Soft Multi Sets. His work has produced 10 peer-reviewed scientific International and National Journal papers. He has published 05 papers in

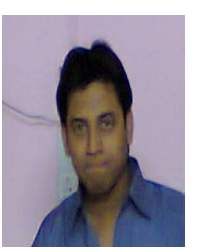
NationalandInternationalConferences. 